\title{
Research on the Evaluation of Urban Science and Technology Innovation Ability in Shaanxi Province Based on BP Neural Network
}

\author{
Li Penglin 1, a , Jia Dong 2,b and Chen Chen ${ }^{3, c}$ \\ 1,2,3 School of Management, Xi'an University of Science and Technology, Xi'an,China \\ alpl@vip.163.com, b957100293@qq.com, c2054477493@qq.com
} Keywords: Scientific and Technological Innovation Ability; Evaluation; BP Neural Network; Shaanxi
Province

\begin{abstract}
Based on the three dimensions of innovation base and supporting ability, technology industrialization ability and brand innovation ability, this paper constructs the index system for the evaluation of urban science and technology innovation ability in Shaanxi province and uses the method of nested BP neural network to carry out empirical evaluation for urban science and technology innovation ability in Shaanxi province, which has obtained the sequence for the technological innovation ability of ten cities in Shaanxi province. The conclusion shows that there is a big gap between the technological innovation ability of various cities in Shaanxi Province. The strongest lies in Guanzhong, the second is in northern Shaanxi, and the weakest in southern Shaanxi. This paper further analyzes the deep reasons for the rankings of scientific and technological innovation ability in the cities of Shaanxi and gives the concrete measures to enhance the innovation ability in Guanzhong, northern Shaanxi and southern Shaanxi on this basis. Guanzhong region wants to optimize the industrial structure, improve the efficiency of resource use and speed up the construction of transformation mechanism; Northern Shaanxi region wants to vigorously develop the tertiary industry to promote industrial restructuring and upgrading; Southern Shaanxi region carries out tilt for high-tech industry in the policy and effectively improve innovation surroundings.
\end{abstract}

\section{Introduction}

Since the development of Western China, Shaanxi province has made great progress in the national economy as an important province in the west of China. However, compared with other eastern regions, the total economic output is still in the middle position in the whole country. According to statistics, in 2016, the total amount of GDP in Shaanxi province ranked fifteenth in the country, which is slightly higher than in the Inner Mongolia Autonomous Region. This is not commensurate with the status of Shaanxi as the large province of education and science and technology in China. The reasons for this situation are multifaceted. There are geographical reasons, resource endowment, economic openness, people's ideas and other reasons. But in these many reasons, the supporting role of technological innovation on economic problems is undoubtedly a key factor. In fact, in the "Evaluation Report of Chinese Regional Innovation Capability" made by the Ministry of science and technology, the performance of Shaanxi Province is not outstanding. Since 2011, it has been ranked in the middle position. In 2015, it ranked 14 in the whole country. The ability of innovation of science and technology is difficult to achieve greater support and promoted role for economy. Therefore, to revitalize the economy of Shaanxi Province, improving the ability of scientific and technological innovation is imperative. If we want to enhance the innovation ability, we must first make scientific evaluation for the technological innovation capability of various cities in Shaanxi province. Only through scientific evaluation, we can clear the advantages and disadvantages of various cities, and develop targeted policy measures through the excavation of its deep causes, which fundamentally enhance the overall development level of Shaanxi's science and technology and promote the healthy development of economy.

Because scientific and technological innovation plays an important supporting role in economic development as the primary productive force, the research results in this field are quite fruitful. The 
current research mainly focuses on the following two aspects: the first is the establishment of evaluation index system; the second is the selection of evaluation methods. In the construction of index system, Tang Yanzhao ${ }^{[1]}$ refers to the sustainable development, science and technology strength and other index systems and construct the evaluation index system of scientific and technological innovation ability from six aspects, such as knowledge innovation capability, the technological innovation capability of enterprise, knowledge flow, knowledge resources, scientific and technological innovation environment and scientific and technological innovation performance. Based on the construction for the index system of national innovation ability, Qiao Zhangfeng [2] constructs the evaluation index system of urban science and technology innovation ability from the four aspects of innovation input, innovation output, innovation support, and innovation environment. Zhang Zhongliang ${ }^{[3]}$ proposes the five elements for the core elements of innovation ability and constructs the evaluation index system of urban science and technology innovation ability from the five aspects of talent structure, technical architecture, industrial structure, institutional structure and social structure. What's more, each structure designs measurement index from the two angles of capital and structure. Based on the application background of urban innovation capability, Du Juan ${ }^{[4]}$ constructs the evaluation index system for the innovation capability of key cities by considering the two stages of input and output. In the selection of evaluation methods, Tang Yanzhao uses fuzzy mathematics method to create a fuzzy comprehensive evaluation model and carries out evaluation for the scientific and technological innovation ability of Guangdong province. Hou Renyong ${ }^{[5]}$ carries out empirical analysis for scientific and technological innovation ability in China by using the principal component analysis method. Li Baizhou ${ }^{[6]}$ uses the rough set method to carry out attribute constraints for the evaluation index of regional scientific and technological innovation ability. Then, through the introduction of entropy model, the quantitative and qualitative mixed problem is integrated into the unified research entirety, and the evaluation model of regionally scientific and technological innovation ability is established. Bi Liangliang ${ }^{[7]}$ uses the evaluation method for the combination of factor analysis and hierarchical clustering analysis to evaluate the technological innovation capability in Yangtze River Delta region, which provides practical basis for the realization of regional innovation and integration for Yangtze River Delta region and the promotion for the development of regional innovation ability. Du Juan divides innovation process into two sub-stages, builds the analysis model of data envelopment and carries out in the evaluation of scientific and technological innovation ability for $52 \mathrm{key}$ cities in China. Tao Xuefe ${ }^{[8]}$ elaborates the relevant concepts of urban science and technology innovation, draws on the index system of science and technology competitiveness and designs the index system of urban science and technology innovation ability. Furthermore, he uses the analytic hierarchy process to empower the indexes in order to carry out evaluation and analysis for key cities in China.

Because each index affects each other, the evaluation of scientific and technological innovation ability is a typical nonlinear problem. In addition, because the sample quantity normally used for evaluation is insufficient, this problem is a typical small sample problem at the same time. Therefore, if we use component analysis, data envelopment analysis, clustering analysis, and other traditional analysis methods to carry out evaluation, which will lead to the greater error between evaluation results and actual situation. In other words, these traditional methods are not appropriate in this problem for the evaluation of technological innovation capacity. The artificial neural network method, as a classic tool for dealing with nonlinear problems, has great advantages in dealing with small sample problems. Therefore, this paper attempts to evaluate the technological innovation ability of cities by establishing BP neural network model.

\section{The construction of index system}

Whether the index system of urban scientific and technological innovation ability is reasonable or not directly affects the quality of evaluation results. In the process of studying urban scientific and technological innovation ability, our scholars construct different evaluation index system. Among them, the "Urban innovation report in China" of Zhou Tianyong and Kuang Jianwei editor constructs the evaluation index system to evaluate urban scientific and technological innovation ability from the 
three dimensions of innovation base and supporting ability, technology industrialization ability and brand innovation ability. This set of index system has stronger practicability and strong pertinence, which is used by many researchers. When this paper constructs the index system, it mainly refers to the "Urban innovation report in China", "Innovation index in China" and "Regional innovation capability report in China". Taking into account the availability and quantification of data, the index of urban comprehensive visibility is deleted, and the following evaluation index system was formed at last, which is shown as table 1.

Table 1 The evaluation system of urban scientific and technological innovation ability

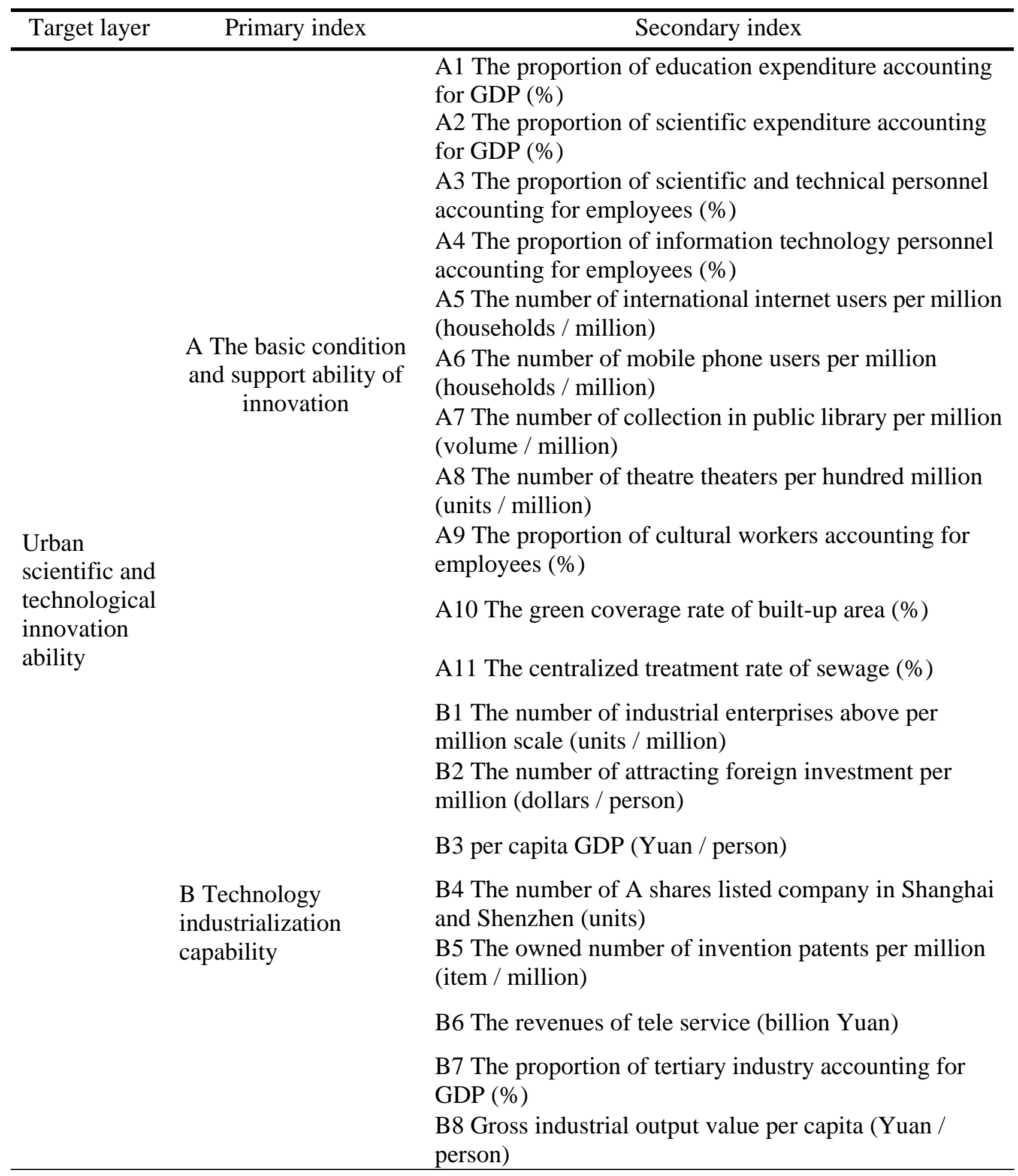


Table 1 to be continued

\section{C1 The number of well-known trademarks (units)}

C Brand innovation capability
C2 The number of A level scenic spots (units)

C3 The number of key cultural relics protection units in the whole country (units)

The basic conditions and support ability of innovation. The basic conditions and support ability of innovation refers to the supporting ability of social basic conditions for innovation, and these basic conditions include a city's policy conditions, personnel protection, information level, cultural atmosphere, sustainable development capacity, etc. The basic conditions and supporting ability of innovation are the important parameters of necessary basic and supporting ability to drive the development of urban scientific and technological innovation ability, which includes specifically the 11 secondary indicators of proportion of education expenditure accounting for GDP, the proportion of science expenditure accounting for GDP, the proportion of science and technology personnel accounting for employees, which represents with A1, A2, ..., A11. Among them, A1 and A2 measure the policy conditions of city; A3 and A4 embodies the talent protection. The proportion of science and information technology personnel accounting for employees characterizes the workers' knowledge level of city, and knowledge level is the decisive factor of innovation; A5 and A6 reflects the information level of city, the application scope of Internet, which is the important parameter of characterizing the scientific and technological progress of city. Moreover, the country put forward the "Internet +" strategy, so the role of Internet for innovation and support greatly enhances. The two indicators are set for this purpose; A7, A8 and A9 embody the ability of cultural support. Cultural support not only can provide cultural atmosphere and inclusive innovation environment for innovation, but it also can provide cultural infrastructure for innovation; A10 and A11 embodies the sustainable development capacity of city. Sustainable development capacity index is an important indicator of reflecting urban development potential, and good ecological environment is one of the important conditions to retain high-tech talent.

Technological industrialization capability. The ability of technological industrialization is the ability of science and technology to transform into productive forces. The capability of technological industrialization reflects the extent, scale and capacity of innovation ability, which specifically includes 8 secondary indicators, such as the number of industrial enterprises above per million scale, the number of attracting foreign investment per million people, which can represent with B1 and $\mathrm{B} 2, \ldots, \mathrm{B} 8$. Among them, the B1 to B5 reflects the industrial conditions of city, and B1 and B2 are the indicator to measure the status of enterprises. As an important component of urban innovation, enterprises can bring more innovation for city and will provide more platforms for urban innovation and development. B3 is the measurement of the city's ability to attract foreign investment. At this stage, foreign capital not only brings the direct benefits of capital and employment, but it also brings the advantages of technology and management. B4 reflects the per capita value of annual economic growth. Only certain economic base can it support the input and output of innovation.B5 reflects the patent situation, and the patent is the performance of innovation. B6, B7 and B8 reflect the industrial structure. Among them, B6 reflects the extent to which modern electronic information technology is used in innovation, and B7 reflects the state and situation of economic restructuring adjustment. The expansion of tertiary industry reflects the development of national economy towards benign and harmonious, which is conducive to the development of urban innovation. B8 is the indicator to directly reflect innovation ability.

Brand innovation ability.Brand innovation ability is to give brand elements to create value. Through technology, quality, business model and enterprise innovation and cultural innovation, it can enhance brand vitality and improve brand competitiveness. Brand innovation ability is the index group to directly reflect the long-term innovation of city, which specifically includes the three secondary indicators of the number of well-known trademarks, A-level scenic spots and the number 
of key cultural relics protection units. It can represent with $\mathrm{C} 1, \mathrm{C} 2$, and $\mathrm{C} 3$. Among them, $\mathrm{C} 1 \mathrm{reflects}$ the results of urban scientific and technological innovation. Enterprise is the basic cell of urban scientific and technological innovation. The brand effect created in the process of inheritance and development is the intangible asset of enterprise and the crystallization of innovation achievement. $\mathrm{C} 2$ is an important indicator of attracting the residents of city. C3 is the important cultural asset of city, and it has a comprehensive influence on foreign capital and humanity. C2 and C3 are important supporting indicators for urban innovation and cultural heritage.

\section{BP artificial neural network method overview}

Artificial Neural Network ${ }^{[9]}$ (ANN) is a network formed by extensive interconnection of a large number of processing units (Neurons) based on the mathematical model of biological principle, and it mainly processes the actual information by simulating the structure and function of the human nervous system, and the handling process of reflection.

The basic principles.BP artificial neural network is a more mature and widely used feed forward neural network. Its structure consists of an input layer, one or more hidden layers and one output layer. The layers are composed of several neurons. The relationship between the output value of a node and the input value is determined by the function and threshold, and the neurons can achieve any non-linear mapping between input and the output. According to the basic principle of neural network, this paper designs a BP neural network evaluation model based on the second-class index of urban science and technology innovation capability, as shown in Figure 1.

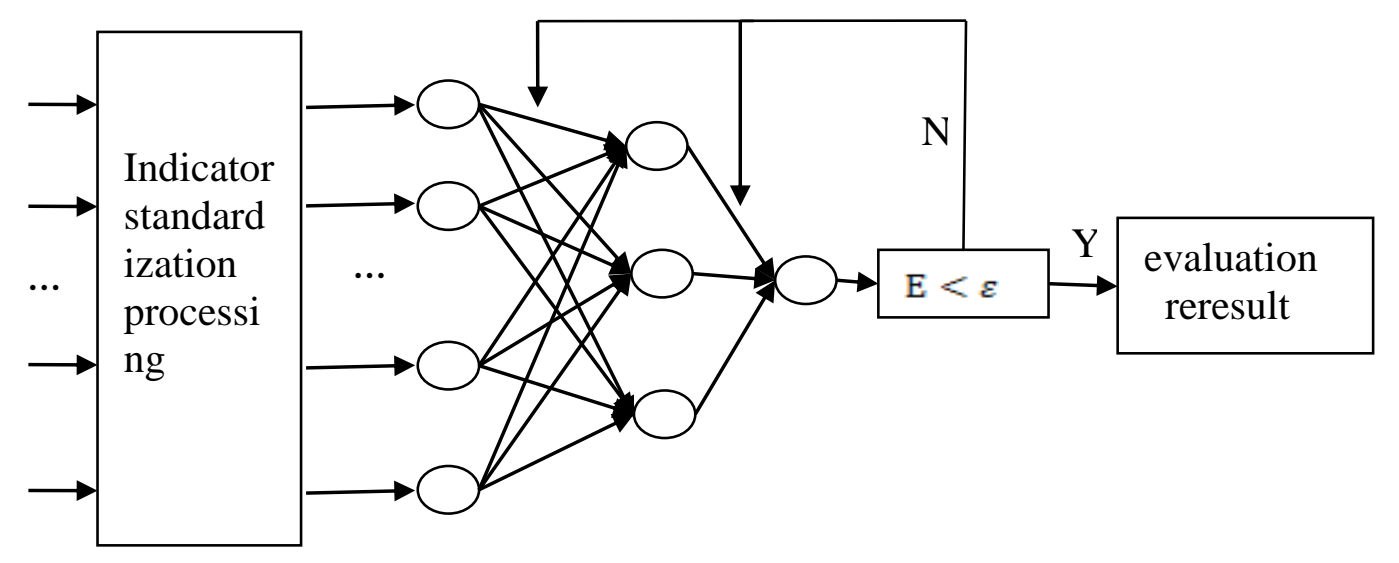

Index layer Input layer Hidden layer Output layer

Fig.1 BP Neural Network Evaluation Model

BP artificial neural network configuration.BP artificial neural network configuration includes four aspects: the determination of the network layer, the determination of the network input layer node, the determination of the number of intermediate implicit layer node, and the determination of the network output layer node. The first is the determination of the number of the network layer. In 1989, Robert Hecht-Nielson ${ }^{[10]}$ proposed a three-dimensional BP network to complete any n-dimensional to m-dimensional mapping and demonstrated it. Therefore, the BP neural network established in this paper is a three-layer network, which consists of an input layer, an intermediate hidden layer and an output layer. The second is the determination of the network input layer node. The number of input nodes corresponds to the number of evaluation indicators. The number of the network input layer node is determined by evaluating the number of specific indicators in the index system. The third is the determination of the number of intermediate hidden layer node. The selection of hidden layer nodes is related to the accuracy and learning efficiency of the whole BP network. It is necessary to take into account the accuracy of the BP network, but also take the network learning methods into 
consideration, but there are no theoretical guidance on the determination of the number of hidden layer nodes, several common empirical formula can be adopted: $\mathrm{i}=\sqrt{\mathrm{nm}}, \mathrm{i}=\log _{2} \mathrm{n}$. Among them, $\mathrm{m}$ is the number of output nodes and $\mathrm{n}$ is the number of input nodes. The paper uses the stepwise regression analysis method and the significance test of the parameters to dynamically remove some linearly related neurons, and finally determines the number of nodes (i) contained in the hidden layer. The fourth is the determination of the network output layer node. The number of output layer nodes corresponds to the number of network output results.

\section{Evaluation network construction}

At the beginning of this paper, all the second-class indicators are proposed as the input layer of the network, and the scientific and technological innovation ability score is used as the output layer. However, due to the excessive number of second-class indicators, the number of samples that can be leant is too small, so the network generalization ability trained is poor. Therefore, this paper uses the nested BP neural network, and establishes the evaluation system which includes three first class indexes: innovation basic condition and support ability, technology industrialization ability and brand innovation ability. First, the second-class indicators correspond to the first-class indicators are used as the input layer, and the output layer is the comprehensive score of the first-class indicators. Then, the score of the first-class indicators is used as the new network input layer, and the output layer is the comprehensive score of the city's scientific and technological innovation ability. Through the use of nested BP neural network, we can not only get the comprehensive score of urban innovation ability, but also can analyze the urban innovation ability according to the score of the city's first-class indicators, and the network has strong generalization ability.

Construction of innovation basic condition and support ability subnetwork .The indicators of innovation basic condition and support ability subnetwork are the foundation of driving the development of urban science and technology innovation ability and important parameters of support ability, including 11 second-class indicators. According to the evaluation results of prefecture-level city innovation basic conditions and support ability in China Urban Innovation Capability Report 2015 Edition, 50 cities are selected as learning samples of BP neural network. The indicator date of sample cities comes from the China Urban Statistical Yearbook 2015 and the city's local statistical yearbook, statistical bulletin and so on.

According to the BP neural network construction method, the network is set as follows: the number of nodes in the input layer is 11 , the number of hidden neurons is 5 , and the number of output nodes is 1 . In order to make the indicators in the whole evaluation system are comparable, the indicators must be dealt with and standardized, and transformed into unit-less variables, and then the 11 second-class indicators of the 50 sample cities processed are used as the training sample inputs of the neural network model, and the score of innovation basic conditions and support ability in China's Urban Innovation Capability Report is used as the output, using MATLAB for training. The specific code is as follows:

$\mathrm{P}=[11 \times 50] ; \%$ sample index input value is a matrix of 11 rows $\times 50$ columns

$\mathrm{T}=[1 \times 50] ; \%$ expected output value is a matrix of 1 row $\times 50$ columns

Net $=$ newff $(\operatorname{minmax}(\mathrm{P}),[11,5,1],\{$ 'tansig', 'tansig', 'purelin' $\}$, 'trainlm');\% generated network

Net $=$ init (net); \% initial network

Net.trainParam.epochs $=500 ; \%$ sets the maximum number of training sessions

Net.trainParam.goal $=0.00001 ; \%$ sets the training target error

Net.trainParam.lr $=0.03 ; \%$ sets the learning rate

Net.trainParam.mc $=0.5 ; \%$ sets the momentum factor

net $=$ train(net,P,T); \% train network

After the training of the network 67 epochs, the training error reaching the expected error, the network convergence effect is good, as shown in Figure 2. 


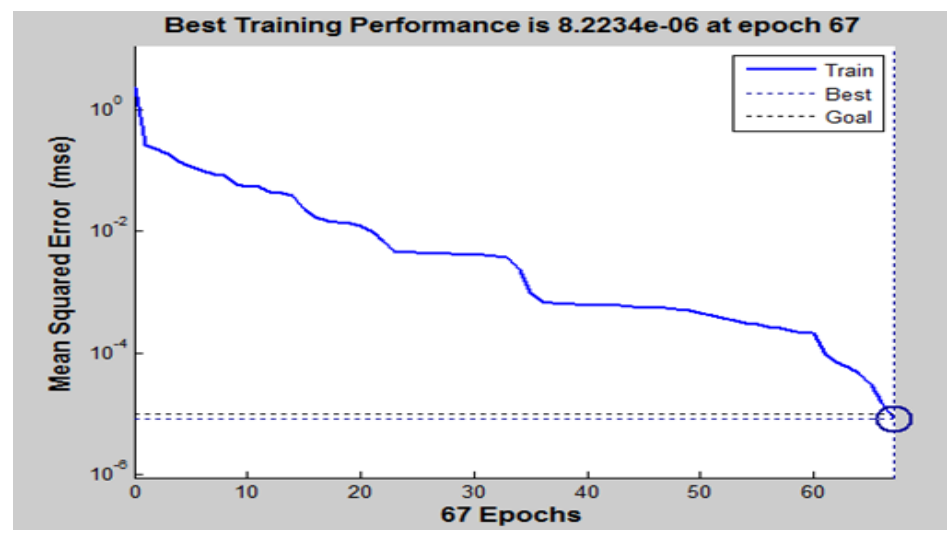

Fig. 2 BP Neural Network Training Results

Adjust the trained model, 7 sets of test data are input into the network model for simulation verification, the model output results shown in Table 2 . From the expected output and the model output, the error is within the acceptable range, the model evaluation results are consistent with the real situation, indicating that the constructed neural network model has strong generalization ability and good applicability.

Table 2 Innovative Basics and Support Subnetwork Test Data Test Results

\begin{tabular}{cccccccc}
\hline Verified sample & 1 & 2 & 3 & 4 & 5 & 6 & 7 \\
\hline Expected output & 96.11 & 79.89 & 73.47 & 69.74 & 67.83 & 62.65 & 60.75 \\
Model output & 95.31 & 78.95 & 72.73 & 69.68 & 67.12 & 63.11 & 60.66 \\
Relative error $(\%)$ & 0.83 & 1.18 & 1.01 & 0.08 & 1.05 & 0.73 & 0.15 \\
\hline
\end{tabular}

Similarly, the technology technical industrialization capacity and brand innovation ability network can also be trained, and the use of trained network to detect the test data, the relative error is within $2 \%$. In view of the length constraint of the paper, it will not repeat them.

The comprehensive evaluation of urban scientific and technological innovation capability Construction of the main BP neural network. The comprehensive score of urban scientific and technological innovation capability is the most intuitive embodiment of urban innovation capability. Based on the aforementioned construction of BP subnetwork, this article takes the scores of three first-level indexes as input and takes the comprehensive score of urban science and technology innovation ability as output, which further constructed the BP neural network model of scientific and technological innovation ability, that is the main BP neural network model of this article. According to the evaluation results of the comprehensive innovation capability of prefecture-level city in The 2015 edition of the urban innovation capability report of China, 20 cities are selected as the learning samples of BP neural networks.

The network settings are as follows: the number of nodes in the input layer is 3, the number of neurons in the hidden layer is 2 and the number of nodes in the output layer is 1 . With the normalization for the data, and then we take the scores of innovation foundation and support ability, technology industrialization capabilities as well as brand innovation ability for the treated 20 samples cities as the training sample input of neural network model, and take the comprehensive scores of scientific and technological innovation ability of samples cities in The urban innovation capability report of China as output, meanwhile, adopting the MATLAB software for training. After 71 steps of training, the training error reaches the expected error, and the convergence effect of the network is 
better. After the verification, the relative error is controlled less than $2 \%$. The test result is shown in table 3 , and the applicability of network is better.

Table 3 The detection result of the total network test data for the evaluation of scientific and technological innovation

\begin{tabular}{cccccccc}
\hline Verified sample & 1 & 2 & 3 & 4 & 5 & 6 & 7 \\
\hline Expected output & 97.33 & 83.95 & 77.13 & 72.82 & 69.17 & 67.77 & 64.95 \\
Model output & 95.79 & 84.34 & 76.83 & 71.99 & 68.63 & 68.21 & 65.47 \\
Relative error $(\%)$ & 1.58 & 0.46 & 0.39 & 1.14 & 0.78 & 0.65 & 0.80 \\
\hline
\end{tabular}

\section{Empirical Evaluation}

Shaanxi province has ten prefecture-level cities, which are traditionally divided into three regions: Guanzhong region includes Xi'an, Baoji, Xianyang, Weinan and Tongchuan; the northern Shaanxi includes Yulin and Yanan; the southern Shaanxi includes Hanzhong, Ankang and Shangluo.

Evaluation results.Utilizing the trained network to evaluate the urban scientific and technological innovation ability of Shaanxi province, the result is shown in table 4.

Table 4 The evaluation results of urban scientific and technological innovation capability in Shaanxi province

\begin{tabular}{c|cccc|cc|ccc}
\hline \multirow{2}{*}{ City } & $\begin{array}{c}\text { The basis and } \\
\text { support of } \\
\text { innovation }\end{array}$ & $\begin{array}{c}\text { Technology } \\
\text { industrialization }\end{array}$ & \multicolumn{2}{c}{$\begin{array}{c}\text { Brand } \\
\text { innovation }\end{array}$} & \multicolumn{2}{c}{$\begin{array}{c}\text { Comprehensive capability } \\
\text { of innovation }\end{array}$} \\
\cline { 2 - 9 } Score & ranking & score & ranking & score & ranking & score & ranking \\
\hline Xi'an & 93.22 & 1 & 97.18 & 1 & 95.13 & 1 & 96.31 & 1 \\
Baoji & 63.35 & 3 & 67.95 & 2 & 64.64 & 3 & 69.17 & 2 \\
Xianyang & 61.55 & 4 & 60.74 & 3 & 62.51 & 4 & 62.44 & 3 \\
Weinan & 59.47 & 5 & 57.54 & 6 & 70.34 & 2 & 61.87 & 4 \\
Yanan & 55.44 & 7 & 59.71 & 4 & 61.40 & 5 & 61.11 & 5 \\
Yulin & 67.15 & 2 & 57.05 & 7 & 56.91 & 9 & 58.85 & 6 \\
Hanzhong & 54.12 & 8 & 56.33 & 8 & 59.01 & 6 & 56.48 & 7 \\
Tongchuan & 58.27 & 6 & 59.48 & 5 & 54.18 & 10 & 55.17 & 8 \\
\hline Shangluo & 52.32 & 9 & 53.69 & 10 & 57.03 & 8 & 54.60 & 9 \\
\hline Ankang & 50.43 & 10 & 54.78 & 9 & 58.43 & 7 & 54.34 & 10 \\
\hline
\end{tabular}

Result analysis and policy proposal.The table 4 shows that there are big disparities among science and technology innovation ability of each city in Shaanxi province. Judging from the ranking of comprehensive capability for innovation of each city, the strongest is Guanzhong region, the northern Shaanxi takes second place and the weakest is the southern Shaanxi, which is consistent with the traditional classification of geographical regions in Shaanxi province.

The scientific and technological innovation capability in Guanzhong area is obviously higher than that in northern Shaanxi and southern Shaanxi. Judging from the comprehensive ranking of innovation ability, the top four are concentrated in the Guanzhong area, which further confirms that Guanzhong area has the strong industrial base. Xi'an ranked first, and its scores are far ahead, it can be 
seen that Xi'an's scientific and technological innovation ability is stronger, which has benefited from more colleges and universities and research institutes in Xi'an. Xi'an should make full use of this advantage, speed up the construction of scientific and technological achievements transformation mechanism so as to improve the scientific and technological innovation ability, and then catch up with the cities in the east with strong scientific and technological innovation ability. The comprehensive ranking of Baoji city is second, which reflects objective facts that Baoji city has many large scale of industrial enterprises and stronger innovative ability. In order to improve the scientific and technological innovation ability, Baoji city should start with optimizing the industrial structure and raising the level of resource utilization. Xianyang and Weinan have similar situation in the development of science and technology, the comprehensive ranking is third and fourth respectively. The capacity of technological industrialization of Xianyang city ranks third, indicating that Xianyang has a high capacity for enterprise innovation in the whole province. The ability of brand innovation in Weinan city ranks second, which has benefited from its paying attention to the enterprise brand, but its capacity of technological industrialization ranks sixth in Shaanxi province, showing that the transformation ability of Weinan city from the science and technology to the productivity remains to be improved. For Xianyang and Weinan city, they could accelerate the transformation of scientific and technological achievements by encouraging enterprises to cooperate with universities and research institutions, at the same time, also should strengthen the introduction and cultivation of technical personnel, introduce technological innovation incentive policies, and safeguard the legitimate rights and interests of technological innovation talents. Although Tongchuan city is in the Guanzhong area, but its overall ranking is relatively backward, which mainly due to its poor brand innovation ability that ranking the penultimate. The Tongchuan municipal government should increase the investment of scientific and technological innovation, and strengthen the construction of basic capabilities for the scientific and technological. At the same time, the government should introduce preferential policies to attract foreign investment, and promote local economic development with the advanced technology that brought about through foreign capital. Guanzhong area should optimize the industrial structure, strive to improve the utilization rate of resources and accelerate the transformation of scientific and technological achievements. Meanwhile, Xi'an should play a leading role to carry out the enterprises cooperation among cities, and share the scientific and technological achievements to further enhance and promote the scientific and technological innovation ability of Guanzhong area.

In northern Shaanxi, Yanan city is ranked fifth place overall, and Yulin city is ranked sixth place overall. The innovation foundation and support ability of Yulin ranked second, which shows that Yulin has a strong sense of innovation. The northern Shaanxi takes the development and utilization of coal, oil and other mineral resources as the main line, due to the coal and oil belong to the resources industries with unsustainablility, so it also should increase the development of the industry with large added value of science and technology to promote the transformation and upgrading of industry. As for Yanan and Yulin city, it is urgent to develop the third industry, the government should support for the third industry on the policy. Yanan should make full use of its advantages with red tourism, vigorously develop the tourism industry and promote the development of related high-tech industries.

The ranking of southern Shaanxi is low, Hanzhong city is ranked seventh place overall, and its sub-items are in a lower middle level. The situation of Shangluo and Ankang is not optimistic, and the rankings of each indicator are relatively low. The shortage of scientific and technological personnel and funds in southern Shaanxi, and the structure of science and technology investment is irrational, which leads to the low scientific and technological innovation ability. In the southern Shaanxi province, we should attach importance to the high-tech industries from the policy, formulate relatively loose and effective economic policies as well as effectively improve the environment of innovation to raise the attraction for foreign funds. While increasing the investment in scientific and technological innovation, the government also should advocate equity participation of the social capital vigorously and share the earnings from scientific and technological innovation. 


\section{Conclusion}

Scientific and technological innovation plays an important supporting role in economic development. Therefore, it would be significant to evaluate the ability of scientific and technological innovation. This article constructed the index system from the three aspects of the innovation basic conditions and support ability, technology industrialization ability and brand innovation ability. Combined with the research problem, this article improved BP neural network, constructed the nested BP neural network and made an empirical evaluation to the technology innovation ability of Shaanxi province. In addition to Xi'an, the science and technology innovation ability of other cities in Shaanxi province is not strong, and these cities are lack of investment in science and technology as well as economic support. Therefore, the cities in Shaanxi province should increase their investment in science and technology to further promote the scientific and technological innovation capability.

\section{Acknowledgements}

Shaanxi Province soft science project "The research on innovation development model in the district of Shaanxi Province(2015KRM085); National natural science foundation project "The theory and policy research on the security management of important energy supply in China under the uncertain conditions" (71273206).

\section{References:}

[1]Tang Yanzhao. A study on the fuzzy comprehensive evaluation model of regional scientific and technological innovation capability and its application-the comprehensive analysis of scientific and technological innovation ability in Guangdong Province in 2001[J]. System Engineering Theory and Practice, 2004, (02): 37-43.

[2]Qiao Zhangfeng, Zhou Zhigang. The evaluation and empirical study on the science and urban technology innovation capability [J]. Journal of Xi' an Electronic and Science University (Social Science Edition), 2011, (03): 62-67.

[3]Zhang Zhongliang, Xing Jingli. A study on the core connotation and measurement of urban science and technology innovation capability [J].Science and Technology Management, 2013, (09): 63-70.

[4] Du Juan, Huo Jiazhen. The evaluation of urban innovation capability in China based on data envelopment analysis [J]. China Management Science, 2014, (06): 85-93.

[5]Hou Renyong, Zhang Lei, Wang Guo. The evaluated index system and empirical study of regional innovation capability [J]. Journal of Wuhan University of Technology (Information and Management Engineering), 2009, (04): 637-641.

[6] Li Bozhou, Su Yi. A study on the evaluation of regional science and technology innovation capability based on improved mutation series [J]. China Soft Science, 2012, (06): 90-101.

[7] Bi Liangliang, Shi Zulin. The evaluation of urban science and technology innovation capability of Yangtze River Delta and the construction of "regional science and technology innovation circle" based on the primary exploration of factor analysis and cluster analysis model [J].Economic Geography, 2008, (06): 946- 951+954.

[8] Tao Xuefei. The evaluated index system and empirical research of urban science and technology innovation comprehensive ability [J]. Economic Geography, 2013, (10): 16-19.

[9] Han Liqun. Artificial neural network [M]. Beijing: Beijing University of Posts and Telecommunications Press, 2006. 
[10] Du Dong, Pang Qinghua, Wu Yan. Modern comprehensive evaluation method and case selection [M]. Beijing: Tsinghua University Press, 2008. 